\title{
Asymptotic behavior of the warm inflation scenario with viscous pressure
}

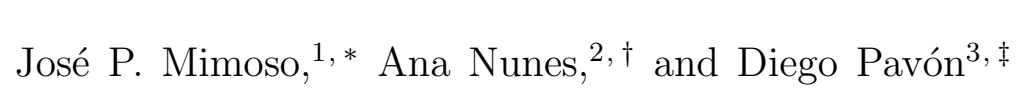 \\ ${ }^{1}$ Department of Physics, Faculdade de Ciências da Universidade de Lisboa, \\ and Centro de Física Teórica e Computacional da Universidade de Lisboa, \\ Av. Prof. Gama Pinto 2, P-1649-003 Lisboa, Portugal \\ ${ }^{2}$ Department of Physics, Faculdade de Ciências da Universidade de Lisboa, \\ and Centro de Física Teórica e Computacional da Universidade de Lisboa, \\ Av. Prof. Gama Pinto 2, P-1649-003 Lisboa, Portugal \\ ${ }^{3}$ Departamento de Física, Universidad Autónoma de Barcelona, Facultad de Ciencias \\ 08193 Bellaterra (Barcelona) Spain
}

(Dated: November 16, 2018)

\begin{abstract}
We analyze the dynamics of models of warm inflation with general dissipative effects. We consider phenomenological terms both for the inflaton decay rate and for viscous effects within matter. We provide a classification of the asymptotic behavior of these models and show that the existence of a late-time scaling regime depends not only on an asymptotic behavior of the scalar field potential, but also on an appropriate asymptotic behavior of the inflaton decay rate. There are scaling solutions whenever the latter evolves to become proportional to the Hubble rate of expansion regardless of the steepness of the scalar field exponential potential. We show from thermodynamic arguments that the scaling regime is associated to a power-law dependence of the matter-radiation temperature on the scale factor, which allows a mild variation of the temperature of the matter/radiation fluid. We also show that the late time contribution of the dissipative terms alleviates the depletion of matter, and increases the duration of inflation.

PACS numbers: $98.80 . \mathrm{Cq}, 47.75+\mathrm{f}$
\end{abstract}

Keywords:

*Electronic address: jpmimoso@cii.fc.ul.pt

${ }^{\dagger}$ Electronic address: anunes@lmc.fc.ul.pt 
${ }^{\ddagger}$ Electronic address: diego.pavon@uab.es 


\section{INTRODUCTION}

There is a widespread belief that our Universe, or at least a sufficiently large part of it causally connected to us, experienced an early period of accelerated expansion, called inflation. This happened before the primordial nucleosynthesis era could take place and likely after the Planck period. Many inflationary scenarios have been proposed over the years (see 1] and references therein). Most of them rely on the dynamics of a self-interacting scalar field (the "inflaton"), whose potential overwhelms all other forms of energy during the relevant period. They generally share the unsatisfactory feature of driving the Universe to such a super-cooled state that it becomes necessary to introduce an ad hoc mechanism -termed "reheating" - in order to raise the temperature of the universe to levels compatible with primordial nucleosynthesis. Therefore this reheating phase appears as a subsequent, separate stage mainly justified by the need to recover from the extreme effects of inflation 2] during which a rather elaborate process of multifield parametric resonances followed by particle production [3, 4, 5] takes place.

As an alternative some authors have looked for inflationary scenarios leading the universe to a moderate temperature state at the end of the superluminal stage so that the reheating phase could be dispensed with altogether. It was advocated that this can be accomplished by coupling the inflaton to the matter fields in such a way that the decrease in the energy density of the latter during inflation is somewhat compensated by the decay of the inflaton into radiation and particles with mass. This would happen when the inflaton rolls down its potential, but keeping the combined pressure of the inflaton and radiation negative enough to have acceleration. This kind of scenario, known as "warm inflation" as the radiation temperature never drops dramatically, was first proposed by Berera [6, 7]. It now rests on solid grounds since it has been forcefully argued in a series of papers that indeed the inflaton can decay during the slow-roll (see, e.g. [8, 9, 10] and references therein). Besides, this scenario has other advantages, namely: $(i)$ the slow-roll condition $\dot{\phi}^{2} \ll V(\phi)$ can be fulfilled for steeper potentials, $(i i)$ the density perturbations generated by thermal fluctuations may be larger than those of quantum origin [11, 12, 13], and (iii) it may provide a very useful mechanism for baryogenesis [14].

To simplify the study of the dynamics of warm inflation, previous works treated the particles created in the decay of the inflaton purely as radiation, thereby ignoring the existence 
of particles with mass in the decay fluid. Here, we will go a step beyond by taking into account the presence of of particles with mass as part of the decay products, and give a hydrodynamical description of the mixture of massless and non-massless particles by an overall fluid with equation of state $p=(\gamma-1) \rho$, where the adiabatic index $\gamma$ is bounded by $1 \leq \gamma \leq 2$

On very general grounds, this fluid is expected to have a negative dissipative pressure, $\Pi$, that somewhat quantifies the departure of the fluid from thermodynamical equilibrium, which we will consider to be small but still significant. This viscous pressure arises quite naturally via two different mechanisms, namely: $(i)$ the inter-particle interactions [15], and (ii) the decay of particles within the matter fluid [16].

A well known example of mechanism $(i)$ of prime cosmological interest is the radiative fluid, a mixture of massless and non-massless particles, as it plays an essential role in the description of the matter-radiation decoupling in the standard cosmological model [17, 18, 19].

A sizeable viscous pressure also arises spontaneously in mixtures of different particles species, or of the same species but with different energies -a typical instance in laboratory physics is the Maxwell-Boltzmann gas [20]. One may think of $\Pi$ as the internal "friction" that sets in as a consequence of the diverse cooling rates in the expanding mixture, something to be expected in the matter fluid originated by the decay of the inflaton.

As for mechanism $(i i)$, it is well known that the decay of particles within a fluid can be formally described by a bulk dissipative pressure $\Pi$. This is only natural because the decay is an entropy-producing scalar phenomenon associated with the spontaneous enlargement of the phase space (we use the word "scalar" in the sense of irreversible thermodynamics) and the bulk viscous pressure is also a scalar entropy-producing agent. There is an ample body of literature on the cosmological applications of this analogy -see e.g. [16], [21]. In the case of warm inflation, it is natural to expect that, at least, some species of particles directly produced by the decay of the inflaton will, in turn, decay into other, lighter species. In this connection, it has been proposed that the inflaton may first decay into a heavy boson $\chi$ which subsequently decays in two light fermions $\psi_{d}[22]$. This is an obvious source of entropy, and therefore it can be modelled by a dissipative bulk pressure $\Pi$.

Our purpose in this paper is to generalize the usual warm inflationary scenario by introducing the novel elements mentioned above, namely the decay of the scalar field into a 
fluid of adiabatic index $\gamma$ rather than just radiation, and especially the dissipative pressure of this fluid, irrespective of the underlying mechanism. We will not dwell on the difficult question of the quantum, non-equilibrium thermodynamical problem underlying warm inflation [8, 23, 24, 25, 26, 27, 28], but rather take a phenomenological approach similar to that considered in several works [29, 30, 31, 32, 33] (which can be traced back to the early studies of inflation [34]). Instead of adopting a model building viewpoint and looking for the implications of specific assumptions, we aim at identifying typical features of models that yield interesting asymptotic behavior. We resort to a qualitative analysis of the corresponding autonomous system of differential equations using the approach developed in [35] that allows the consideration of arbitrary scalar field potentials. We will characterize the implications of allowing for various forms of the rate of decay of the scalar field, as well as for various forms for the dissipative pressure. We consider, for instance, models with scalar field potentials displaying an asymptotic exponential behavior. These arise naturally in generalized theories of gravity emerging in the low-energy limits of unification proposals such as super-gravity theories or string theories [36]. On the one hand, after the dimensional reduction to an effective 4-dimensional space-time and the subsequent representation of the theories in the so-called Einstein frame typical polynomial potentials become exponential [37, 38]. On the other hand, the theories are then characterised by the existence of a scalar field that couples to all non-radiation fields, with the coupling depending, in general, on the scalar field. The simplest example of these features can be found in the so-called non-minimal coupling theories. We provide a classification of the relevant global dynamical features of the cosmological model associated with those possible choices. A limited account of some of the results of the present work was reported in [39].

One question we address is whether non-trivial scaling solutions [33, 35, 40, 41] (hereafter simply termed scaling solutions) exist, i.e., solutions where the ratio of energies involving the matter fluid and scalar field keep a constant ratio. Another class of solutions refered in the literature as having a scaling asymptotic behavior are those for which both the energy density of the scalar field and that of the matter fluid decay with different power laws of the scale factor of the universe [40, 45, 46]. In this latter case one of the components eventually dominates and thus the ratio of their energy densities becomes evanescent, in clear contrast to the case of the non-trivial scaling solutions. We shall term these solutions as trivial scaling solutions to contrast them with the previous ones sometimes dubbed tracker solutions. The 
trivial case arises in association with scalar field potentials of a power-law type and, as we shall see, they occur when the scalar field decays have the the same type of time-dependences as those required by the (non-trivial, tracking) scaling solutions.

One of the reasons why non-trivial scaling solutions are important is that they provide an asymptotic stationary regime for the energy transfer between the scalar field and radiation. This stationary (sometimes termed "quasi-static") regime is an assumption in the standard treatment of warm inflation [11] to evaluate the temperature of matter in the final stages. On the other hand, introducing this class of solutions in the kinetic analysis of interacting fluids [42, 43] leads to an alternative to the usual $\Gamma \gg 3 H$ case, generalizing the example of Ref. [44] where temperature of the matter (radiation) bath is nearly constant.

We show that this class of scaling behavior depends not only on the asymptotic form of the inflaton [35], but also on having an appropriate time-dependent rate for the scalar field decay. The additional consideration of bulk viscosity, besides being a natural ingredient in models with one or more matter components as well as in models with inter-particle decays, facilitates the Universe to have a late time de Sitter expansion.

An outline of this work is as follows. Section II studies the model underlying the original idea of the warm inflation proposal, namely the model in which the inflaton field decays into matter during inflation thus avoiding the need for the post-inflationary reheating. This decay is characterized by a rate $\Gamma$ which we shall initially assume to be a constant. Our results though will argue in favour of a varying $\Gamma$ and we shall thus consider the case where $\Gamma \propto H$. This yields late time scaling solutions whenever the scalar field potentials asymptotes to an exponential behavior. This happens regardless of the slope of the potential. Subsequently, section IIII, analyses more realistic models where a bulk viscous pressure term $\Pi$ is also present in the equation of state of matter. We first envisage the usual form $\Pi=-3 \zeta H$ for that pressure and, subsequently, analyse a general model with both a varying rate of decay and a general form for the bulk viscosity $\Pi=-3 \zeta \rho^{\alpha} H^{\beta}$, where $2 \alpha+\beta=2$ on dimensional grounds. Finally, section IV provides a discussion of our results. 


\section{THE DYNAMICS OF WARM INFLATION}

\section{A. Warm inflation with constant $\Gamma$}

We consider a spatially flat Friedmann-Robertson-Walker universe filled with a selfinteracting scalar field and a perfect fluid consisting of a mixture of matter and radiation,

such that the former decays into the latter at some constant rate $\Gamma$. For the time being we ignore the dissipative pressure. We also neglect radiative corrections to the inflaton potential [12, 24]. The corresponding system of equations reads

$$
\begin{aligned}
3 H^{2} & =\rho+\frac{\dot{\phi}^{2}}{2}+V(\phi), \\
\dot{H} & =-\frac{1}{2}\left(\dot{\phi}^{2}+\gamma \rho\right), \\
\ddot{\phi} & =-(3 H+\Gamma) \dot{\phi}-V^{\prime}(\phi),
\end{aligned}
$$

where here and throughout we use units in which $8 \pi G=c=1$. The first two are Einstein's equations, the third describes the decay of the inflaton. From these, it follows the energy balance for the matter fluid,

$$
\dot{\rho}=-3 \gamma H \rho+\Gamma \dot{\phi}^{2} .
$$

As usual $H \equiv \dot{a} / a$ denotes the Hubble factor.

To cast the corresponding autonomous system of four differential equations it is expedient to introduce the set of normalized variables

$$
\begin{aligned}
x^{2} & =\frac{\dot{\phi}^{2}}{6 H^{2}} \\
y^{2} & =\frac{V(\phi)}{3 H^{2}} \\
r & =\frac{\Gamma}{3 H},
\end{aligned}
$$

along with the new time variable $N=\ln a$. Thus we get

$$
\begin{aligned}
& x^{\prime}=x(Q-3(1+r))-W(\phi) y^{2}, \\
& y^{\prime}=(Q+W(\phi) x) y,
\end{aligned}
$$




$$
\begin{aligned}
& r^{\prime}=r Q, \\
& \phi^{\prime}=\sqrt{6} x,
\end{aligned}
$$

where a prime means derivative with respect to $N$, and the definitions

$$
W(\phi)=\sqrt{\frac{3}{2}}\left(\frac{\partial_{\phi} V}{V}\right)
$$

and

$$
Q=\frac{3}{2}\left[2 x^{2}+\gamma\left(1-x^{2}-y^{2}\right)\right],
$$

as well as $\rho /\left(3 H^{2}\right)=1-x^{2}-y^{2}$ were used. Equation (111) was first considered in [35], and is crucial for the consideration of general potentials $V(\phi)$ besides the particular case of the exponential potential. The function $Q$ defined by Eq. (13) is related to the deceleration parameter $q=-\ddot{a} a / \dot{a}^{2}$ by $Q=1+q$.

The special case where $r=0$, naturally, corresponds to the absence of interaction between the scalar field and the perfect fluid, and it is an invariant manifold of the dynamical system (8) - (111). It is appropriate to refer here its major features in order to better appreciate the implications of the decay of the scalar field (see Table 【).

We distinguish the fixed points of the system into those occurring for finite values of $\phi$ and those associated with the asymptotic limit, $\phi \rightarrow \infty$. In the former case, i.e., for finite $\phi=\phi_{*}$, the fixed points always require the vanishing of the kinetic energy of the scalar field $(x=0)$. They are located at the origin $(x=y=0)$, and at $(x=0, y=1)$, on the frontier of the phase space domain $x^{2}+y^{2}=1$, which is an invariant manifold. For $x=0, y=0$, the potential must have a vanishing critical point at $\phi_{*}$, a case that cannot be dealt with the variables in use, but it is well-know that if $\phi_{*}$ is a minimum at the origin, then it is a stable point and the scale factor evolves as $a(t) \propto t^{2 /(3 \gamma)}[46$, 50]. The fixed points on $x^{2}+y^{2}=1$ are given by $x=0, y=1$ and require that $W=0$. This means that they can only occur in association with extrema of the potential. Their stability is defined by the sign of $W^{\prime}\left(\phi_{*}\right)$, where $\phi_{*}$ is the value of $\phi$ where $V^{\prime}(\phi)$ (and hence $W$ ) vanishes. When $V$ has a non-vanishing minimum and, hence $W^{\prime}>0$, the critical point is a stable node. When $V$ has a maximum and, hence $W^{\prime}<0$, we have a saddle point (an unstable fixed point). These fixed points correspond to the de Sitter exponential behavior and are accompanied by the depletion of the matter component $(\rho=0)$. 
To study the critical points that occur at $\phi \rightarrow \infty$ (which we shall label $\phi_{\infty}$ ), we carry out the regularization produced by the change of variable $\psi=1 / \phi$. Then Eq. (11) becomes

$$
\psi^{\prime}=-\sqrt{6} x \psi^{2},
$$

and the critical points correspond to either to $x=0$, as previously seen, or $\psi=0$. The $\phi_{\infty}$ critical points depend on the asymptotic behavior of $V(\phi)$ [35]. If $V(\phi)$ exhibits some non-vanishing asymptotic value we have again $x=0, y=1$ corresponding to a cosmological constant and, hence, to a de Sitter late-time behavior. If $V(\phi)$ asymptotes towards the exponential potential, say $V \propto e^{-\lambda \phi}$, with $\lambda$ constant, there are several possible fixed values dependent on the ratio between $\lambda^{2}$ and $\gamma$ (see, for instance, [48] for details). There are unstable fixed points on the invariant manifolds bounding the phase-space domain for all possible choices of both $W=-\sqrt{3 / 2} \lambda$ and $\gamma$, namely: $(i)$ a matter dominated solution at $x=0$ and $y=0$, which is a saddle and corresponds to $a(t) \propto t^{2 /(3 \gamma)}$, (ii) two solutions dominated by the scalar field kinetic energy at $x= \pm 1$ and $y=0$ which are either unstable nodes or saddles, and correspond to the stiff behavior $a(t) \propto t^{1 / 3}, \phi_{\infty}(t) \sim \ln t^{K_{0}}$, where $K_{0}$ is an arbitrary constant defining the scalar field initial velocity. There is another fixed point on the $x^{2}+y^{2}=1$ boundary representing a scalar field dominated solution, when $W^{2}<9$. This fixed point is stable when $W^{2}<9 \gamma / 2$, and unstable otherwise (saddle). Thus for $W^{2}>9 \gamma / 2$ (i.e., $\lambda^{2}>3 \gamma$ ), there is a stable fixed point in the interior of the phase space domain. This latter point corresponds to scaling behavior between the matter and scalar field energy-densities [38, 40, 41, 47, 48, 49]. This attractor solution is characterized by $a(t) \propto t^{2 / 3 \gamma}$ and $\phi-\phi_{0}=\ln t^{ \pm 2 / \lambda}$.

There are also trivial scaling solutions for which $\rho_{\phi} \propto a^{-n}$ and $\rho \propto a^{-m}$, where $n>m$ are positive constants, when [46]

$$
V(\phi)=A^{2}\left(1-\frac{n}{m}\right)^{2}\left(\frac{6-n}{2 n}\right)\left(\frac{\phi}{A}\right)^{\varpi}
$$

where

$$
\varpi=\frac{2 n}{n-m} .
$$

Coming back to the model that includes the interaction and thus letting $r$ be nonvanishing, we immediately see from Eq. (10) that, along the $r$-direction, all the points are singular points if and only if $Q=0$. For finite values of $\phi$, as $x=0$ at the fixed points, this requires once more $y^{2}=1$ so that the singular points are associated with $\rho=0$, i.e., with the 
depletion of the matter component. Moreover, as in the $r=0$ case, these singular points are extrema of the potential $V(\phi)$. They correspond to a de Sitter behavior $\left(a(t) \propto e^{\sqrt{V\left(\phi_{0}\right) / 3} t}\right.$, $\phi=\phi_{0}$ constant) and are either stable or unstable depending on the extremum being a minimum $\left(W^{\prime}>0\right)$ or a maximum $\left(W^{\prime}<0\right)$. In fact, at the singular points corresponding to extrema of the potential $V(\phi)$, the eigenvalues found in the linear stability analysis are

$$
\begin{aligned}
\mu_{y} & =-3 \gamma \\
\mu_{x, \phi} & =-\frac{3(1+r)}{2}\left[1 \pm \sqrt{1-\frac{4 \sqrt{6} W^{\prime}\left(\phi_{0}\right)}{9(1+r)^{2}}}\right]
\end{aligned}
$$

On the other hand, we no longer have fixed points at $x=0, y=0$ (unless $\gamma=0$ which corresponds to the perfect fluid being a cosmological constant). This happens because the system then evolves along the $r$-axis towards $r \rightarrow \infty$, a behavior that can only be prevented by the existence of a positive minimum of the potential $V(\phi)$. At $\phi \rightarrow \infty$ the system does not exhibit scaling solutions anymore. The only fixed points allowed in this asymptotic limit are those associated with a non-vanishing, asymptotically flat potential, which thus corresponds to the de Sitter exponential behavior.

Accelerated expansion corresponds to the region of the phase space where $Q<1$, so that

$$
3 \gamma y^{2}-3(2-\gamma) x^{2}>3 \gamma-2
$$

This condition does not carry any dependence either on $r$ or $\phi$. Thus we may restrict our discussion to a $(x, y)$ projection of the phase space. The condition (19) defines for $1<\gamma<2$ the region between the upper branch of the hyperbolae $3 \gamma y^{2}-3(2-\gamma) x^{2}=(3 \gamma-2)$ and the boundary $x^{2}+y^{2}=1$ of the phase space domain (see Figure 1). The asymptotes of the hyperbolae are $y= \pm \sqrt{(2-\gamma) / \gamma}$, and we see that, as $\gamma$ increases, the inflationary region becomes progressively smaller. In fact the region shrinks vertically towards the $x=0, y=1$ point and it reduces to it in the limit case of $\gamma=2$.

In Ref. 11] the end of inflation is given by the condition $\rho_{\phi} \simeq \rho_{\gamma}$ and this event is associated with the beginning of the matter (radiation) domination. As it becomes apparent from the above discussion, the condition for the end of inflation, $Q=1$, is more general and does not strictly require matter domination. Taylor and Berera's condition [11] corresponds to the end of slow-roll inflation (i.e., $\dot{\phi}^{2} \simeq 0 \simeq x$ ) and is extended, in the present study, to general $\gamma$-fluids as

$$
\rho_{m} \simeq \frac{2}{3 \gamma-2} \rho_{\phi}
$$


The independence on $r$ of the size of inflationary region should not though be understood as the interaction having no effect on inflation. From Eqs (17) and (18) we see that the eigenvalues of the linearized system at the fixed points carry a dependence on $r$ which is such that it renders the minima of the potential more stable and the maxima less unstable (as if the potential became shallower). Thus the transfer of energy from the scalar field to the perfect fluid favors inflation in that the system spends a longer time in the neighborhood of the extrema of the potential. This is exactly what is meant to happen in the warm inflation scenario where it is assumed that slow roll holds and argued that $r$ allows for steeper potentials than those required in its absence. As discussed in [11], it is a simple matter to see that the slow-roll condition on $\dot{\phi}$

$$
\dot{\phi}=-\frac{V^{\prime}}{3 H(1+r)} \simeq-\frac{V^{\prime}}{3 r H},
$$

is easier to satisfy if the scalar field decays, that is, if $r>1$ and much easier if $r \gg 1$.

The fact that $r$ increases indefinitely in the present model is a consequence of its definition, and merely translates the fact that, unless the system is trapped at a non-vanishing minimum of $V(\phi), H$ decreases towards zero. Since this is a direct result of assuming a constant $\Gamma$, we consider in the next section a more appropriate model where $\Gamma$ decreases as the Universe's expansion proceeds.

\section{B. Warm inflation with $\Gamma \propto H$}

We assume that $\Gamma_{\phi}=3 \Gamma_{*} H$ where $\Gamma_{*}$ is a dimensionless, positive constant. As $H$ is expected to be a non-increasing function of time in an expanding universe, this is a simple choice for the time dependence of $\Gamma_{\phi}$ such that the decays have a stage of maximum intensity (when inflation occurs) followed by a progressive attenuation until it vanishes altogether.

Now $r=\Gamma_{*}$ is a constant parameter and the dynamical system reduces to the three equations

$$
\begin{aligned}
x^{\prime} & =x[Q-3(1+r)]-W(\phi) y^{2}, \\
y^{\prime} & =[Q+W(\phi) x] y, \\
\phi^{\prime} & =\sqrt{6} x
\end{aligned}
$$

where $Q$ is still given by Eq. (13) We see that these equations are analogous to those of the $r=0$ case of the previous section with a different coefficient on the linear term in $x$ 
of Eq. (22). Thus the basic qualitative dynamical features remain the same as those found for that model (see Table III). The decay of the scalar field though introduces two major consequences worthing to be emphasized.

Besides the fact that the origin $x=0, y=0$ is again a fixed point associated with the vanishing of the scalar field's energy and, hence, corresponds to the matter domination, the interaction given by a non-vanishing $r$ has the relevant effect (already found in the constant $\Gamma$ model) that the stability of the minima is reinforced and that the maxima become less unstable. Moreover, the scalar field decay prevents the existence of the fixed points at $x= \pm 1, y=0$, that would correspond to a behavior completely dominated by the scalar field's kinetic energy (and which was, therefore, associated with a stiff behavior in the $r=0$ case).

The other major effect of the interaction arises when we look for fixed points with $x^{2}+y^{2}<$ 1 at $\phi \rightarrow \infty$. Now, we find that there are always attracting scaling solutions for potentials that have an asymptotic exponential behavior, that is, for potentials for which $W \rightarrow$ const when $\phi \rightarrow \infty$ [35]. Moreover, this happens independently of the steepness of the late time exponential behavior which is a remarkable effect of the present model for the transfer of energy from the scalar field to the matter.

Indeed the latter solutions are given by the roots of the system of equations

$$
(u-1)\left(u-\frac{a}{b}\right)-r u=0
$$

and

$$
\cos ^{2} \theta=\frac{\lambda^{2}}{6(1+r)^{2}} \xi^{2}
$$

where $u=\xi^{2}$ and $\theta$ are polar coordinates, $x=\xi \cos \theta$ and $y=\xi \sin \theta$, and where we have defined

$$
\begin{aligned}
& a=\frac{\gamma}{2} \\
& b=\frac{\lambda^{2}}{6(1+r)^{2}}
\end{aligned}
$$

as well as $W_{\infty}=-\sqrt{3 / 2} \lambda$. It is a simple matter to conclude that the effect of a non-vanishing $r$ is such that equations (25) and (26) always have one non-vanishing root within the range of allowed values for $\xi$ and for $\cos \theta$, and hence there are scaling solutions regardless of the ratio between $\lambda^{2}$ and $3 \gamma$. Furthermore linear stability analysis shows that the scaling solutions 
are stable. It is important nevertheless to remark that although scaling solutions emerge for any ratio of $\lambda^{2} / \gamma$, the way that the $\gamma_{\text {eff }}$ index associated with the effective equation of state inducing the power-law scaling behavior is shifted from the corresponding $\gamma$ value of the scaling solutions in the absence of decays depend on $\lambda^{2}$ being larger or smaller than $\gamma$.

Assuming the potential to be asymptotically given by $V \propto e^{-\lambda \phi}$, the latter solutions are $a(t) \propto t^{A}, \phi-\phi_{0}=\ln t^{ \pm 2 / \lambda}$, where $A$ is given in implicit form by

$$
3 \gamma\left(A-\frac{2}{3 \gamma}\right)\left(A-\frac{2}{\lambda^{2}}(1+r)\right)-\frac{4 r}{\lambda^{2}}=0 .
$$

Notice that we can define $\gamma_{\text {eff }}=2 /(3 A)$. A linear expansion about $r=0$ in the neighborhood of the scaling solution (for $\lambda^{2} \neq 3 \gamma$ ) yields

$$
A=\frac{2}{3 \gamma}\left[1+\frac{\frac{2}{\lambda^{2}}}{\left(\frac{2}{3 \gamma}-\frac{2}{\lambda^{2}}\right)} r\right]
$$

when $\lambda^{2} \neq 3 \gamma$. So the decays have the effect of increasing (resp. decreasing) the scale factor rate of expansion with regard to the $r=0$ case if $\lambda^{2}>3 \gamma$ (resp. $\lambda^{2}<3 \gamma$ ). In particular we can see that the scaling behavior can be inflationary, for cases where this would not happen in the absence of decays. For instance, taking $\gamma=4 / 3$ and $\lambda^{2}>4$, the condition for the scaling solution to be inflationary is $1+r>\lambda^{2} / 4>1$. Thus, in this model, the solutions yield endless power-law inflation even for a modest scalar field decay, provided that the asymptotic behavior of the potential is steep enough, i.e., $\lambda^{2}>3 \gamma(>4$ in the present example).

Naturally, besides the scaling solutions, there can also be fixed points corresponding to de Sitter behavior $x=0, y=1$, whenever the scalar field potential exhibits an asymptotic, non-vanishing constant value. However, when the potential is asymptotically exponential, that there are no fixed points on the boundary $x^{2}+y^{2}=1$ at $\phi_{\infty}$ in contrast to what happens in the $r=0$ case.

From a thermodynamical viewpoint, the above scaling solutions are particularly interesting. In a universe with two components, it can be shown [42, 43] that the temperature of each of the components satisfies the equation

$$
\frac{\dot{T}_{i}}{T_{i}}=-3 \frac{\dot{a}}{a}\left(1-\frac{\Gamma_{i}}{3 H}\right) \frac{\partial p_{i}}{\partial \rho_{i}}+\frac{n_{i} \dot{s}_{i}}{\partial \rho_{i} / \partial T_{i}},
$$

where $i=1$ or $2, n_{i}$ denotes the number density of particles of the $i$-species, $\Gamma_{i}$ their rate of decay, and $T_{i}$ the temperature of this component. In the important case of particle 
production with constant entropy per particle, $\dot{s}_{i}=0$, we also have $\left(\rho_{1}+p_{1}\right) \Gamma_{1}=-\left(\rho_{2}+\right.$ $\left.p_{2}\right) \Gamma_{2}$. Thus, taking the first component to be the matter/radiation fluid and the second to be the inflaton scalar field, we have

$$
\Gamma_{\phi}=\frac{(\rho+p)}{\dot{\phi}^{2}} \Gamma_{m / r} \propto \Gamma_{m / r}
$$

As $(\rho+p) / \dot{\phi}^{2}=\gamma\left(1-x^{2}-y^{2}\right) / 2 x^{2}$ is a constant in the scaling solutions, $\Gamma_{\phi}=3 r H$, with $r$ a constant, implies $\Gamma_{m / r}=3 \sigma H$, where $\sigma$ is another constant that depends both on $r$ and on the location of the scaling solution. This yields a temperature of the matter/radiation component evolving as a power-law $T \propto a^{-3(\gamma-1)(1-\sigma)}$. Thus for $\sigma$ close to 1 , the temperature of the matter/radiation remains quasi-static, whereas for $\sigma>1$ (resp. $\sigma<1$ ), it increases (resp. decreases). Notice also that for $\sigma \simeq 0$, we recover the temperature law for perfect fluids without dissipative effects. Provided we guarantee enough inflation, $r$ need not be very large (contrary to what is usually assumed to facilitate slow-rolling). Indeed, the temperature of the radiation at the end of inflation is

$$
T_{\text {end }}=T_{\text {beginning }} e^{-N(1-\sigma)} \text {, }
$$

where $N$ is the number of e-foldings. Thus, a value of sigma lower but sufficiently close to 1 has the potential to avoid a serious decrease of the temperature of the universe. As

$$
\sigma=\frac{3 x_{*}^{2}}{2\left(1-x_{*}^{2}-y_{*}^{2}\right)} r
$$

at the scaling solutions, we see that $r$ need not be very large to ensure that $\sigma \sim 1$.

Trivial scaling solutions generalizing those given Eq. (15) in the $r=0$ case, arise in these models for scalar field potentials of the form

$$
V(\phi)=A^{2}\left(1-\frac{n}{m}\right)^{2}\left(\frac{6(1+r)-n}{2 n}\right)\left(\frac{\phi}{A}\right)^{\varpi},
$$

where $\varpi$ is still given by Eq. (16) and $A=A_{r=0} / \sqrt{1+r}$. For the potential to be positive one also requires $0<m<n<6(1+r)$. The only difference with respect to the $r=0$ case lies in the dependence on $r$ of the constant factor multitplying $\phi^{\varpi}$, and translates the fact that there is now a different distribution of the scalar field energy density between its kinetic and potential parts. Indeed, we have that

$$
V(\phi)=\left[\frac{6(1+r)}{n}-1\right] \dot{\phi}^{2}
$$


which shows that the extra damping of the kinetic energy part when $r \neq 0$, as expected. As in the $r=0$ case, the possible emergence of the trivial scaling behavior in association with monomial potentials (that might or not be part of double wells) happens when the matter fluid is already dominating and is thus of a lesser importance in the context of warm inflation.

\section{WARM INFLATION WITH BULK VISCOSITY}

One of the main purposes of the present work is to assess the implications for warm inflation of the presence of a viscous pressure, $\Pi$, in the matter component, so that the total fluid pressure is $p=(\gamma-1) \rho+\Pi$. We may assume the expression $\Pi=-3 \zeta H$ which, albeit some causality caveats, is the simplest one may think of and has been widely considered in the literature [51, 52, 53]. If the mixture of massive particles and radiation is taken as a radiative fluid, it is admissible to adopt $\zeta \propto \rho_{\gamma} \tau$, where $\tau$ denotes the relaxation time of the

dissipative process. For the hydrodynamic approach to apply the condition $t_{\text {col }} H<1$ should be fulfilled. Since $\tau \propto t_{\text {col }}$ (a reasonable assumption) and the most obvious time parameter in this description is $H^{-1}$, one concludes that $\Pi \simeq-\beta \rho_{\gamma}$, with $0<\beta<1$.

This modifies the field equations (2) and (4) which now read

$$
\begin{aligned}
\dot{H} & =-\frac{\dot{\phi}^{2}+\gamma \rho+\Pi}{2} \\
\dot{\rho} & =-3\left(\gamma+\frac{\Pi}{\rho}\right) H \rho+\Gamma \dot{\phi}^{2},
\end{aligned}
$$

while equations (11) and (3) remain in place and thus the dynamical system is now

$$
\begin{aligned}
x^{\prime} & =x[Q-3(1+r)]-W(\phi) y^{2}, \\
y^{\prime} & =[Q+W(\phi) x] y, \\
\chi^{\prime} & =\chi\left[\left(\frac{\Pi^{\prime}}{\Pi}\right)+2 Q\right], \\
r^{\prime} & =r Q \\
\phi^{\prime} & =\sqrt{6} x,
\end{aligned}
$$

where

$$
\chi=\Pi /\left(3 H^{2}\right),
$$


and

$$
Q=\frac{3}{2}\left[2 x^{2}+\gamma\left(1-x^{2}-y^{2}\right)+\chi\right] .
$$

Apart from raising the order of the system, the main difference with regard to the previous cases lies in the modification introduced in $Q$. In fact the bulk viscosity term contributes an additional term to it, and this changes the properties of some of the fixed points of the warm inflation model. These implications naturally depend on the functional form of $\Pi$, and next we consider some specific choices.

\section{A. Warm inflation with bulk viscosity $\Pi=-3 \zeta H$}

Our first choice is the "classical" assumption already mentioned, $\Pi=-3 \zeta H$, where $\zeta$ is a positive constant ensuring that the second law of thermodynamics holds. We also assume that $\Gamma_{\phi}$ is constant as in Subsection

Given the definition of $\chi$ in the dynamical system (39] 43), we see that $\chi=-\zeta / H \propto r$. Therefore, defining the constant $\bar{r}=-\Gamma / 3 \zeta$ so that we have $r=\bar{r} \chi$, not only Eq. (41) considerably simplifies, but also we do not need the $r$ equation (42). The resulting dynamical system is

$$
\begin{aligned}
x^{\prime} & =x[Q-3(1+\bar{r} \chi)]-W(\phi) y^{2}, \\
y^{\prime} & =[Q+W(\phi) x] y, \\
\chi^{\prime} & =\chi Q \\
\phi^{\prime} & =\sqrt{6} x .
\end{aligned}
$$

We see from Eq. (48) that this system has fixed points with vanishing bulk viscosity, $\chi=0$, which send us back to the cases already studied in section III. The novel situations, however, arise when the fixed points occur with $\chi \neq 0$, which requires $Q=0$ (meaning that $H=H_{*}$ is constant).

For finite values of $\phi$, say at $\phi_{*}$, the fixed points are defined by $x_{*}=0, W\left(\phi_{*}\right) y_{*}^{2}=0$. So we have a fixed point at $x_{*}=0, y_{*}=0, \chi=\chi_{*}, \phi=\phi_{*}$, where $\chi_{*}$ is given by

$$
\chi_{*}=-\gamma
$$

Being associated with the vanishing of both $\dot{\phi}$ and $V(\phi)$, this is, remarkably, a matter dominated de Sitter solution. It is stable if the potential has a vanishing minimum. Alternatively, 
we have a line of fixed points given by $x_{*}=0$ and by $\gamma\left(1-y_{*}^{2}\right)=-\chi_{*}$, in accordance to the $Q_{*}=0$ condition. This solution corresponds again to a de Sitter exponential behavior and has the remarkable feature that the energy densities of the scalar field and of the matter remain in a fixed proportion. It arises in association with an extremum of the potential $V(\phi)$ and its stability depends on the extremum being a maximum or a minimum, the maximum being unstable and the minimum stable. The presence of $r=\bar{r} \chi$ will again contribute to render the minima more stable and the maxima less unstable, as already found in the study of the cases devoid of viscous pressure.

It is appropriate to emphasize that, for these classes of fixed points, the matter energy density is not depleted by the inflationary behavior. This is due, of course, to the wellknown fact that the bulk viscosity contributes a negative pressure and induces inflationary behavior.

Now, regarding the fixed points arising at $\phi \rightarrow \infty$, the situation is similar to that considered in Subsection IA. In fact the functional forms adopted both by $\Gamma$ and $\Pi$ prevent the existence of scaling solutions. The only fixed points associated with the asymptotic behavior of $V(\phi)$ are analogous to those at finite $\phi$. If $V(\phi)$ has a vanishing asymptotic value, we have again both the $x=0$ and $y=0, \chi=-\gamma$ de Sitter solutions dominated by matter, and when $V(\phi)$ exhibits a non-vanishing asymptotic value at infinity, we have the $x=0$, $\chi=-\gamma\left(1-y^{2}\right)$ de Sitter solutions characterized by a constant ratio between the energies of matter and of the scalar field. Incidentally, one may remark that now the parameter $r$ that represents the decay of the scalar field takes a fixed value determined by the $r=\bar{r} \chi$ relation at the fixed points.

We conclude this Section commenting that if we were to assume the type of decay considered in Subsection IB i.e., a constant $r$, the features of the dynamical system would be the same as in the case just considered. This means that the remarkable effect we found there that attractor scaling solutions would always emerge when the potential has an asymptotic exponential behavior is destroyed by the addition of bulk viscosity of the type $\Pi=-3 \zeta H$.

\section{B. Warm inflation with general bulk viscosity and decay terms}

We now extend our previous analysis to ascertain the implications of more general functional dependences of both $\Gamma_{\phi}$ and $\Pi$. 
We assume, quite generally, $\Gamma_{\phi}=\tilde{\Gamma}(\phi) H^{\delta}$ and $\Pi=-3 \zeta \rho^{\alpha} H^{\beta}$, where $\delta>0, \zeta, \alpha$ and $\beta$ are constants and moreover $2 \alpha+\beta-2=0$ on dimensional grounds. The latter condition on the parameters $\alpha$ and $\beta$ implies that the dimensionless variable $\chi$ becomes $\chi=-3^{\alpha} \zeta\left(\rho / 3 H^{2}\right)^{\alpha}$ and, hence, it reduces to $\chi=-3^{\alpha} \zeta\left(1-x^{2}-y^{2}\right)^{\alpha}$, since, as previously, we still have $\rho / 3 H^{2}=1-x^{2}-y^{2}$. The $\delta$ free parameter controls how fast $\Gamma_{\phi}$ decreases with decreasing $H$ during slow-roll inflation (see Eq.(137)). Moreover, for $\delta>1, r$ decreases with decreasing $H$, and that for $\delta<1$ it increases.

The dynamical system is 4 -dimensional and reads

$$
\begin{aligned}
x^{\prime} & =x(Q-3(1+r))-W(\phi) y^{2}, \\
y^{\prime} & =(Q+W(\phi) x) y, \\
r^{\prime} & =r\left[\sqrt{6}\left(\frac{\partial_{\phi} \tilde{\Gamma}}{\tilde{\Gamma}}\right) x+Q(1-\delta)\right] \\
\phi^{\prime} & =\sqrt{6} x,
\end{aligned}
$$

where $Q$ is now given by

$$
Q=\frac{3}{2}\left[2 x^{2}+\gamma\left(1-x^{2}-y^{2}\right)-3^{\alpha} \zeta\left(1-x^{2}-y^{2}\right)^{\alpha}\right] .
$$

In what follows it seems reasonable to further assume that $0<\alpha<1$ so that $\beta>0$ which amounts to having a bulk viscosity pressure whose importance diminishes with the expansion and with the dilution of matter.

Inspection of Eqs. (51 55.5) shows that it becomes possible to avoid the restrictive $Q=0$ condition previously found in the $\Gamma=$ constant and $\Pi=-3 \zeta H$ models that implied a de Sitter behavior at the fixed points. We have now a wider range of possibilities (our results for the scaling solutions are summarised in Table ஹII).

At finite values of $\phi$ we find a line of fixed points $x=y=r=0$, and another line of fixed points characterized by $x=0, \phi=\phi_{0},\left(1-y^{2}\right)^{1-\alpha}=3^{\alpha} \zeta / \gamma$ and any value of $r$. In the latter case $\phi_{0}$ is the value of $\phi$ at an extremum of $V(\phi)$, i.e., where $W\left(\phi_{0}\right)=0$, and in order to guarantee that $y^{2} \leq 1$ we require that

$$
3^{\alpha} \zeta<\gamma
$$

(Notice that this amounts to having $\rho+p+\Pi>0$, hence ensuring $\dot{\rho}<0$, regardless of the ratio $\rho /\left(3 H^{2}\right)$. It is, thus, a condition akin to the usual weak energy condition). 
The linear stability analysis shows that the singular points $x=y=r=0$ corresponding to matter domination are unstable. In fact the eigenvalues are

$$
\begin{aligned}
\lambda_{\phi} & =0 \\
\lambda_{r} & =\frac{3}{2}(1-\delta)\left(\gamma-3^{\alpha} \zeta\right) \\
\lambda_{y} & =\frac{3}{2}\left(\gamma-3^{\alpha} \zeta\right)>0 \\
\lambda_{x} & =3\left(\frac{\gamma-3^{\alpha} \zeta}{2}-(1+r)\right),
\end{aligned}
$$

and we see that $\lambda_{y}$ is positive.

Regarding the line of singular points with $r \neq 0$, linear stability analysis reveals that, besides the vanishing eigenvalue associated with $r\left(\lambda_{r}=0\right)$, the sability is once more determined by the nature of the extremum of $V(\phi)$. Indeed, the eigenvalue corresponding to $y$ is

$$
\lambda_{y}=2 \gamma y_{*}^{2}(\alpha-1)<0, \quad \text { when } \alpha<1 \text {, }
$$

where $y_{*}$ is a solution of $\left(1-y_{*}^{2}\right)^{1-\alpha}=3^{\alpha} \zeta / \gamma$, and the eigenvalues along $x$ and $\phi$ are given by

$$
\lambda_{x, \phi}=-\frac{3(1+r)}{2} \pm \frac{1}{2} \sqrt{9(1+r)^{2}-4 \sqrt{6} y_{*}^{2} W^{\prime}\left(\phi_{0}\right)} .
$$

We see from the latter equation that $\lambda_{x, \phi}>0$ requires that $W^{\prime}\left(\phi_{*}\right)<0$, that is a maximum at $V\left(\phi_{*}\right)$. Otherwise, in the case of a minimum of $V(\phi)$, we have either a stable node (when $\left.0<W^{\prime}\left(\phi_{0}\right)<9(1+r)^{2} /\left(4 \sqrt{6} y_{*}^{2}\right)\right)$ or a stable sink $\left(\right.$ when $\left.W^{\prime}\left(\phi_{0}\right)>9(1+r)^{2} /\left(4 \sqrt{6} y_{*}^{2}\right)>0\right)$.

As is well-known, some authors have resorted to the cooperative action of many scalar fields -the so-called "c inflation" -, both in cool inflation [54] and in warm inflation [55], to get a sufficiently flat effective potential capable of driving power-law accelerated expansion. Here we note that this can be achieved with just a single inflationary field provided the dissipative bulk viscosity is not ignored. Indeed, this can be seen from from Eq. (62). An increase in the parameter $r$ as well as a decrease of $y_{*}$ induce an effective reduction of the steepness of the potential at the maxima and a greater stability of the minima. Since $y_{*}^{2}=1-\left(3^{\alpha} \zeta / \gamma\right)^{1 /(1-\alpha)}$, a decrease in $y_{*}$ translates an increase in $\zeta$ within the admissible range $\left(3^{\alpha} \zeta / \gamma<1\right)$. The net effect is that the system spends a longer time in the neighborhood of a fixed point associated with a maximum of the potential (alternatively, the minima become more stable). This is helpful for setting the conditions for slow-roll inflation. In fact, this alleviates the need for a large rate of decay of the scalar field. We just need $\left[3(1+r) / y_{*}\right]^{2}>1$. 
At $\phi \rightarrow \infty$, labelled $\phi_{\infty}$, Eq. (53) shows that we may have the usual fixed points corresponding to a non-vanishing, flat asymptotic asymptotic behavior of the potential (late-time approach to a cosmological constant) if $Q=0, \Gamma^{\prime} / \Gamma=0$ and $\Pi^{\prime} / \Pi=0$ simultaneously. However, we also find asymptotic scaling behavior in the case $W(\phi)$ approaches an exponential behavior $\left(W\left(\phi_{\infty}\right)=-\sqrt{3 / 2} \lambda\right.$, with constant $\left.\lambda>0\right)$, provided

$$
W(\phi)=\frac{\sqrt{6}}{1-\delta}\left(\frac{\partial_{\phi} \tilde{\Gamma}}{\tilde{\Gamma}}\right)
$$

which amounts to having, at $\phi \rightarrow \infty, \tilde{\Gamma}(\phi) \propto(V(\phi))^{\frac{1-\delta}{2}}$ and $\tilde{\Gamma}$ must be asymptotically exponential. Notice that for $\delta=1$ we recover the $\Gamma \propto H$ rate of decay considered in section (IIB). The scaling solutions are then characterized in polar coordinates, $x=\xi \cos \theta$, $y=\xi \sin \theta$, by

$$
\cos \theta_{*}=\frac{\lambda}{\sqrt{6}(1+r)} \sqrt{u}
$$

where $u \equiv \xi^{2}$ is a root of the equation

$$
(1-u)(a-b u)-r b u-\frac{3^{\alpha} \zeta}{2}(1-u)^{\alpha}=0
$$

The quantities $a$ and $b$ were defined above.

We see from Eqs. (64) and (65) that there is always one (and only one) scaling solution, provided the condition (56) holds. (Notice that this was precisely the condition that was required for the existence of fixed points at finite $\phi)$. Indeed, the first two terms of Eq. (65) are a second-order polynomial $P_{2}(u)$ with $P_{2}(0)>0$ and $P_{2}(1)<0$, so that it has one, and only one zero, in that interval $(0,1)$. Thus the addition (subtraction) of the $\frac{3^{\alpha} \zeta}{2}(1-u)^{\alpha}$ has the net effect of making the root of $P_{2}$ approach the origin $u=0$, and the latter remains in the $(0,1)$ interval provided (56) is valid. We also find that the location of the singular points corresponding to the scaling behavior is now closer to $x^{2}+y^{2}=0$, having a smaller $y_{\infty}$ value than in the models without bulk viscosity. Moreover, linear stability analysis reveals that under the conditions (56) and $\alpha<1$ the scaling solutions are stable, i.e., are attractors. These results mean that the late time contribution of the matter component is enhanced by the viscous pressure. This is a most convenient feature for the warm inflation scenario, since it further alleviates the depletion of matter during inflation and the subsequent need for reheating. 
The power law behavior of these solutions is $a \propto t^{A}, \phi \sim \ln t^{2 / \lambda}$ with $A$ given in implicit form by

$$
(3 \gamma A-2)\left[A-\frac{2}{\lambda^{2}}(1+r)\right]=\frac{4}{\lambda^{2}} r+3^{1+\alpha} \zeta A^{2-\alpha}\left[A-\frac{2}{\lambda^{2}}(1+r)\right]^{\alpha}
$$

where $r$ is here the asymptotic value of this parameter at the scaling solution (where, $r \propto$ $y_{*}^{\frac{1-\delta}{2}}$ ). A linear expansion in both $r$ and $\zeta$ in the neighborhood of the scaling solution when $r=0, \zeta=0$, and $\lambda^{2} \neq 3 \gamma$, yields

$$
A(\gamma, \lambda ; r, \zeta) \simeq \frac{2}{3 \gamma}\left[1+\frac{\left(\frac{2}{\lambda^{2}}\right)}{\frac{2}{3 \gamma}-\frac{2}{\lambda^{2}}} r+\frac{3^{1+\alpha}}{2}\left(\frac{2}{3 \gamma}\right)^{2-\alpha}\left(\frac{2}{3 \gamma}-\frac{2}{\lambda^{2}}\right)^{\alpha-1} \zeta\right]
$$

Naturally, these equations reduce to the Eqs. (29) and (30) when $\delta=1$ and $\zeta=0$. As found in subsection IB, it is possible to define in the same manner a $\gamma_{\text {eff }}=2 /(3 A)$. We see that now it might be possible to have $\lambda^{2}<3 \gamma$ if $\alpha$ is, for instance, a rational of the type $\alpha=m /(2 n)$ with $m(<2 n)$ and $n$ integers. However for $\alpha=m /(2 n+1), \lambda^{2}$ can be both larger or smaller than $3 \gamma$, rspectively, yielding $\gamma_{\text {eff }}$ smaller or larger than $\gamma$. In other words, larger or smaller values of $A$ with regard to the case without either decays or bulk viscosity.

It is interesting to look at the modifications of the regions of the phase space that correspond to inflationary behavior arising from the consideration of the viscous pressure. In particular, it is important to assess how they depend on the choice of the parameters. From Figure 2 we see that the size of the inflationary region is larger than in the corresponding models without viscous pressure (models with the same $\gamma$ ). This was expected as the bulk viscosity term amounts to a negative pressure. We also see, in good agreement with this, that the size of the inflationary region decreases with decreasing $\zeta$, as the importance of the viscous pressure diminishes.

We conclude this section by briefly commenting on the trivial scaling solutions. The scalar field potentials that yield trivial scaling solutions when the matter fluid dominates are still given by Eq. (35). The requirement that $r$ be a constant now translates into

$$
\tilde{\Gamma}(\phi) \propto\left[(V(\phi))^{\frac{\delta-1}{2}}\right]^{\frac{2-\varpi}{\varpi}}
$$

and in addition we have a consistency condition

$$
\frac{1}{3}\left(\frac{m-\gamma}{3 \zeta}\right)^{\frac{1}{\alpha-1}}=1
$$


Once again, the inequalities $0<m<n<6(1+r)$ must be satisfied if the potential is to be positive. Moreover, as in the $\Gamma \propto H$ case, these scaling solutions happen when matter dominates so that they are not important for warm inflation.

\section{DISCUSSION AND CONCLUSIONS}

In this work we have analyzed the dynamical implications for the warm inflation scenario of the existence of a viscous pressure in the matter content of the Universe. The dissipative pressure may arise either because the fluid in which the inflaton decays may be treated as a radiative fluid or because the different particles making up the fluid cool a different rates or because the particles in which the inflaton decays experience a subsequent decay in another particles species. We have adopted a phenomenological approach and have classified the asymptotic behavior of models associated with possible choices of the inflaton potential, as well as those arising from various functional dependences both of the rate of decay of the scalar field and of the viscous pressure on the matter/radiation component. In general terms we have considered $\Gamma_{\phi}=\Gamma(\phi) H^{\delta}$, where $\delta$ is a constant, and $\Pi=-3 \zeta \rho^{\alpha} H^{2(1-\alpha)}, \alpha<1$ being a constant.

Relevant asymptotic regimes arise in association with maxima and minima of the inflaton potential, at finite $\phi$, and with the asymptotic exponential behavior of the potential at $\phi_{\infty}$. In the latter case, we have found that the existence of scaling solutions depends on the form of the decay rate of the scalar field. Indeed, a necessary and sufficient condition to have scaling solutions is that the rate of decay $\Gamma_{\phi} \propto \Gamma(\phi) H^{\delta}$ becomes proportional to $H$ and, thus, $\Gamma(\phi)$ is required to become asymptotically exponential, as $\Gamma_{\infty} \propto\left(V_{\infty}\right)^{\frac{\delta-1}{2}}$. In contrast to the scaling solutions found in the models without decays (the $r=0$ models), here we find scaling solutions regardless of the steepness of the potential, that is for any combination of $V^{\prime} / V=-\lambda$ and $\gamma$. However the ratio between these values defines whether the effective value of the $\gamma$-index characterising the scaling behavior, and hence the behavior of the scalar field itself, is larger or smaller than the corresponding value of $\gamma$ for the models without decays. Indeed, $\gamma_{e f f}<\gamma$ when $\lambda^{2}>3 \gamma$ and, conversely, $\gamma_{e f f}>\gamma$ when $\lambda^{2}<3 \gamma$.

Moreover inflation may be facilitated and is of the power-law type. On the one hand, inflationary behavior emerges in association with small values of the $r$ parameter. On the

other hand, the additional presence of bulk viscosity helps in avoiding a difficulty faced by 
the warm inflation scenario that was raised by Yokoyama and Linde [24]. Their argument was that if, on the one hand, to enhance slow-roll and simultaneously avoid the depletion of matter, one should have a sufficiently high rate of decay of the scalar field, on the other hand, this would make inflation stop earlier, since the transfer of energy from the scalar field to matter would make the conditions for the domination of the scalar field cease swiftly.

Overall, the presence of dissipative pressure in the matter component (which arises on very general physical grounds) lends strength to the warm inflationary proposal.

\section{Acknowledgments}

JPM and AN wish to acknowledge the financial support from "Fundação de Ciência e Tecnologia" under the CERN grant POCTI/FNU/49511/2002 and the C.F.T.C. project POCTI/ISFL/2/618, and are grateful to J.A.S. Lima for helpful discussions. DP is grateful to the Centro de Física Teórica e Computacional da Universidade de Lisboa for warm hospitality financial support. This research was partially supported by the old Spanish Ministry of Science and Technology under Grants BFM2003-06033 and the "Direcció General de Recerca de Catalunya" under Grant No. 2001 SGR-00186.

[1] E. W. Kolb and M. S. Turner, The Early Universe (Addison-Wesley, Reading, Massachussetts, 1990); A. R. Liddle and D. Lyth, Cosmological Inflation and Large Scale Structure (Cambridge University Press, Cambridge, 2000); A. Linde, "Particle Physics and Inflationary Cosmology", hep-th/0503203.

[2] A. Berera, "Dissipative dynamics of inflation, in Particles, Strings", and Cosmology, Proceedings of the Eighth International Conference, eds. P. Frampton and J. Ng (Rinton Press, 2001., p.393), hep-ph/0106310.

[3] L. Kofman, A.D. Linde and A.A. Starobinsky, Phys. Rev. D 56:3258 (1997), hep-ph/9704452.

[4] B.A. Bassett, D.I. Kaiser and R. Maartens, Phys. Lett. B, 455:84 (1999).

[5] T. Charters, A. Nunes and J. P. Mimoso, Phys. Rev. D71:083515 (2005), hep-ph/0502053

[6] A. Berera, Phys. Rev. Lett., 75:3218 (1995).

[7] A. Berera and L.Z. Fang, Phys. Rev. Lett., 74:1912 (1995). 
[8] A. Berera and R. O. Ramos, Phys. Rev. D 71:023513 (2005).

[9] L.M. Hall and I.G. Moss, Phys. Rev. D 71:023514 (2005).

[10] M. Bastero-Gil and A. Berera, Phys. Rev. D 71:063515 (2005).

[11] A. Berera, Nucl. Phys. B585:666 (2000); A.N. Taylor and A. Berera, Phys. Rev. D 62:083517 (2000).

[12] L.M.H. Hall, I.G. Moss and A. Berera, Phys. Rev. D 69:083525 (2004), astro-ph/0305015.

[13] S. Gupta et al, Phys. Rev. D 66:043510 (2002).

[14] R.H. Brandenberger and M. Yamaguchi, Phys. Rev. D 68:023505 (2003).

[15] L. Landau and E.M. Lifshitz, Mécanique des Fluides (MIR, Moscou, 1971); K. Huang, Statistical Mechanics (J. Wiley, 1987).

[16] Ya. B. Zel'dovich, Sov. Phys. JETP Lett. 12:307 (1970); J.D. Barrow, Nucl. Phys. B 310:743 (1988).

[17] S. Weinberg, Gravitation and Cosmology, pp. 51-58 (J. Wiley, N.Y., 1972).

[18] N. Udey and W. Israel, Mon. Not. R. Astr. Soc. 199:1137 (1982).

[19] D. Jou and D. Pavón, Astrophys J., 291:447 (1983).

[20] S. Harris, An Introduction to the Theory of Boltzmann Equation (Holt, Reinhart and Winston, N.Y., 1971); C. Cercignani, Theory and Applications of the Boltzmann Equation (Scottish Academic Press, Edinburgh, 1975).

[21] W. Zimdahl and D. Pavón, Phys. Lett. A 176:57 (1993); W. Zimdahl and D. Pavón, Mon. Not. R. Astron. Soc. 266:872 (1994); W. Zimdahl and D. Pavón , Gen. Relativ. Grav. 26:1259 (1994); W. Zimdahl, Mon. Not. R. Astron. Soc. 280:12 (1996), W. Zimdahl, Phys. Rev. D $53: 5483(1996)$.

[22] A. Berera and R.O. Ramos, Phys. Lett. B 567:294 (2003); A. Berera and R.O. Ramos, Phys. Rev. D 71:023513 (2005).

[23] E. Calzetta, B.L. Hu, Phys. Rev. D37:2878 (1988).

[24] J. Yokoyama and A. Linde, Phys. Rev. D 60:083509 (1999).

[25] A.Berera, M. Gleiser, R.O. Ramos, Phys. Rev. D 58:123508 (1998), hep-ph/9803394.

[26] A. Berera, M. Gleiser, R. O. Ramos, Phys. Rev. Lett., 83:264-267 (1999), hep-ph/9809583.

[27] Ian G Moss, Nucl. Phys. B, 631:500 (2002), hep-ph/0103191.

[28] I.D. Lawrie, Phys. Rev. D 66:041702 (2002), hep-ph/0204184.

[29] H.P. de Oliveira and R.O. Ramos, Phys. Rev. D 57:741 (1998), gr-qc/9710093. 
[30] M. Bellini, Phys. Lett. B, 428:31 (1998).

[31] J.M.F. Maia and J.A.S. Lima, Phys. Rev. D 60:101301 (1999), astro-ph/9910568).

[32] J. Yokoyama, K. Sato and H. Kodama, Phys. Lett. B, 196:129 (1987).

[33] A.P. Billyard and A.A. Coley, Phys. Rev. D 61:083503 (2000), astro-ph/9908224

[34] A. Albrecht, P.J. Steinhardt, M.S. Turner and F. Wilczek, Phys. Rev. Lett., 48:1437 (1982).

[35] A. Nunes and J.P. Mimoso, Phys. Lett. B 488:423 (2000).

[36] K.A. Olive, Phys. Rep. 190:308 (1990); M.B. Green, J.H. Schwarz, and E. Witten, Superstring Theory (Cambridge University Press, Cambridge, 1978); A. Salam and E. Sezgin, Phys. Lett B 147:47 (1984).

[37] C. Wetterich, Astron. Astrophys. 301:321 (1995), J.J. Haliwell, Phys. Lett B 185:341 (1987).

[38] A. Nunes, J.P. Mimoso and T.C. Charters, Phys. Rev. D 63:083506 (2001).

[39] J.P. Mimoso, A. Nunes and D. Pavón, "Scaling Behaviour in Warm Inflation", in Proceedings of the AIP Conference Phi in the Sky. The Quest for Cosmological Scalar Fields (Porto, Portugal, 2004).

[40] B. Ratra and P.J.E. Peebles, Phys. Rev. D 37:3406 (1988).

[41] D. Wands, E.J. Copeland and A.R. Liddle, Ann. N. Y. Acad. Sci., 688:647 (1993).

[42] W. Zimdahl, J. Triginer and D. Pavón, Phys. Rev. D 54:6101 (1996).

[43] W. Zimdahl and D. Pavón, Gen. Rel. Grav., 33:791 (2001), astro-ph/0005352.

[44] J.A.S. Lima and J.A. Espichan Carrillo, "Thermodynamic approach to warm inflation", astro-ph/0201168.

[45] I. Zlatev, L. Wang and P. J. Steinhardt, Phys. Rev. D59:123504 (1999).

[46] A. R. Liddle and R. J. Scherrer, Phys. Rev. D59:023509 (1999).

[47] P.G. Ferreira and M. Joyce, Phys. Rev. Lett., 79:4740 (1997).

[48] E.J. Copeland, A.R. Liddle and D. Wands, Phys. Rev. D 57 (1998) 4686.

[49] C. Wetterich, Nucl. Phys. B, 302:668 (1988) .

[50] V.A. Belinski, L.P. Grischuk, Ya. B. Zeldovich and I.M. Khalatnikov, Sov. Phys. JETP, 63:195 (1985).

[51] G. Murphy, Phys. Rev. D 8:423 (1973).

[52] S. Weinberg, Astrophys. J., 168:175 (1971).

[53] V.A. Belinski and I.M. Khalatnikov, Sov. Phys. JETP, 62:195 (1978).

[54] A.R. Liddle, A. Mazumdar, and F.E. Schunck, Phys. Rev. D 58:0661301 (1998); A.A. Coley 
and R.J. van den Hoogen, Phys. Rev. D 62:023517 (2002).

[55] L.P. Chimento, A.S. Jakubi, D. Pavón and N. Zuccalá, Phys. Rev. D 65:083510 (2002). 

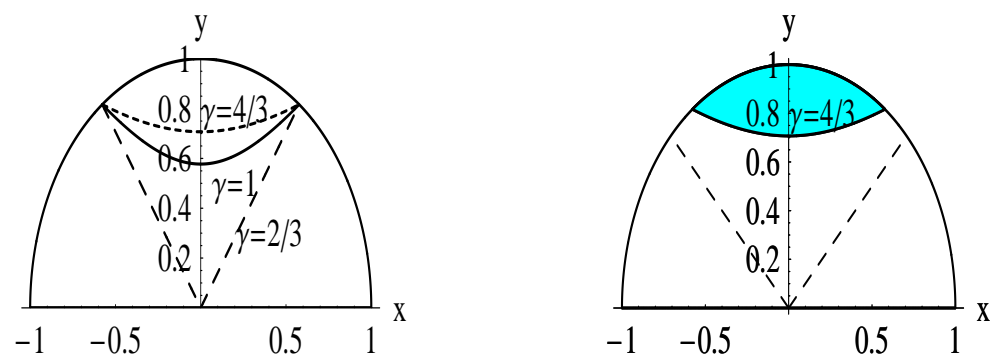

FIG. 1: Inflationary region of the models without bulk viscosity. Inflation occurs in the shaded region between the hyperbole and the boundary of the phase space. The figure on the left depicts the variation of the region with $\gamma$, whereas in the figure on the right we have taken $\gamma=4 / 3$. 


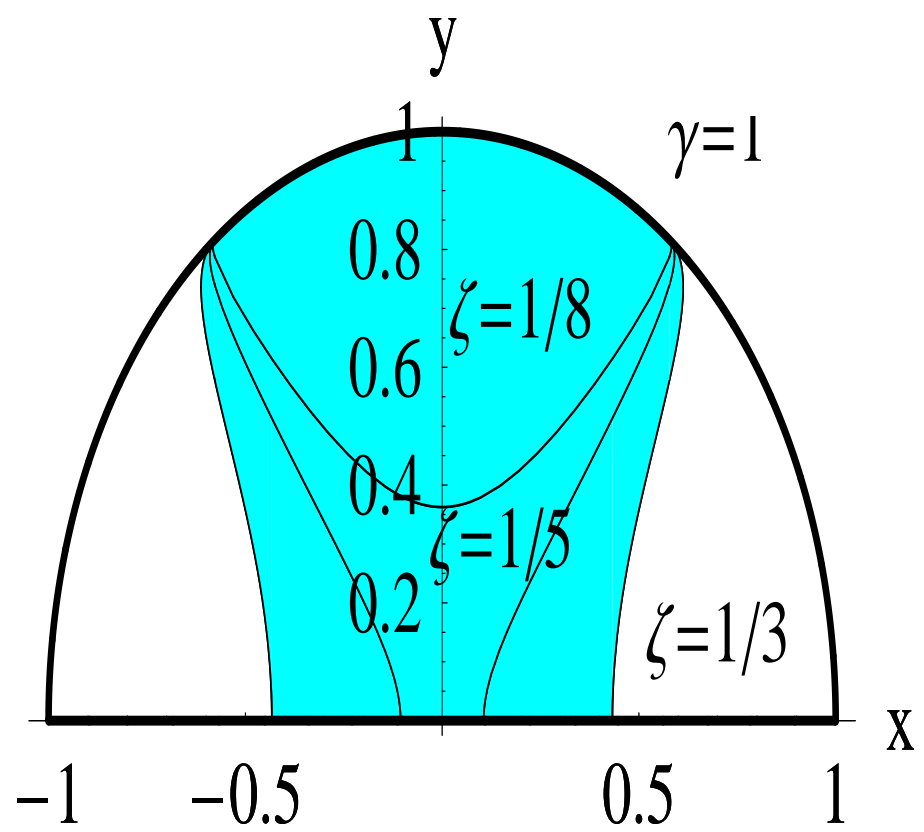

FIG. 2: Inflationary region of the models with bulk viscosity for $\gamma=1$. Inflation occurs in the shaded regions between the border lines and the $x^{2}+y^{2}=1$ boundary of the phase space. The lowest line corresponds to $\zeta=1 / 3$, the intermediate line to $\zeta=1 / 5$ and the uppest line to $\zeta=1 / 8$. We see that the size of the inflationary region decreases with $\zeta$. 
TABLE I: The properties of the asymptotic behavior of the $\Gamma=0$ model. In this table, $\mathrm{S}$ stands for stable, U for unstable, MD for matter dominated, SFD for scalar field dominated, Min for minimum, Max for maximum, and Scal. Sol. for scaling solution.

\begin{tabular}{|c|c|c|c|c|c|c|}
\hline $\mathrm{r}$ & $x_{*}$ & $y_{*}$ & $\phi$ & $V(\phi)$ & Eq. state & Stability \\
\hline \multirow{11}{*}{0} & 0 & 0 & $\phi_{*}$ & $V_{*}=0 \operatorname{Min}(V)$ & - & $\mathrm{S} / \mathrm{MD}$ \\
\hline & 0 & 0 & $\phi_{*}$ & $V_{*}=0$ & - & $\mathrm{U} / \mathrm{MD}$ \\
\hline & 0 & 1 & $\phi_{*}$ & $V_{*} \neq 0 \operatorname{Min}(V)$ & $\gamma_{\phi}=0$ & $\mathrm{~S} / \mathrm{SFD}$ \\
\hline & 0 & 1 & $\phi_{*}$ & $V_{*} \neq 0 \operatorname{Max}(V)$ & $\gamma_{\phi}=0$ & $\mathrm{U} / \mathrm{SFD}$ \\
\hline & 0 & 0 & $\phi_{\infty}$ & $V_{\infty}=0$ & - & $\mathrm{U} / \mathrm{MD}$ \\
\hline & 0 & 1 & $\phi_{\infty}$ & $V_{\infty} \neq 0$ & $\gamma_{\phi}=0$ & $\mathrm{~S} / \mathrm{SFD}$ \\
\hline & \pm 1 & 0 & $\phi_{\infty}$ & $V \sim e^{-\lambda \phi}$ & $\gamma_{\phi}=2$ & U. (saddle)/SFD \\
\hline & $\lambda / \sqrt{6}$ & $\sqrt{1-\lambda^{2} / 6}$ & $\phi_{\infty}$ & $V \sim e^{-\lambda \phi}$ & $\gamma_{\phi}=\lambda^{2} / 3$ & $\mathrm{~S}$ (node)/SFD \\
\hline & $\lambda / \sqrt{6}$ & $\sqrt{1-\lambda^{2} / 6}$ & $\phi_{\infty}$ & $V \sim e^{-\lambda \phi}$ & $\gamma_{\phi}=\lambda^{2} / 3$ & U. (saddle)/SFD \\
\hline & $\sqrt{\frac{3}{2}}\left(\frac{\gamma}{\lambda}\right)$ & $\sqrt{\frac{3(2-\gamma) \gamma}{2 \lambda^{2}}}$ & $\phi_{\infty}$ & $V \sim e^{-\lambda \phi}$ & $\gamma$ & S/Scal. Sol., $3 \gamma<\lambda^{2}<\frac{24 \gamma^{2}}{9 \gamma-2}$ \\
\hline & $\sqrt{\frac{3}{2}}\left(\frac{\gamma}{\lambda}\right)$ & $\sqrt{\frac{3(2-\gamma) \gamma}{2 \lambda^{2}}}$ & $\phi_{\infty}$ & $V \sim e^{-\lambda \phi}$ & $\gamma$ & S/Scal. Sol., $\lambda^{2}>\frac{24 \gamma^{2}}{9 \gamma-2}$ \\
\hline
\end{tabular}


TABLE II: The properties of the asymptotic behavior of the $\Gamma \propto H$ model. In this table, $\mathrm{S}$ stands for stable, U for unstable, MD for matter dominated, SFD for scalar field dominated, Min for minimum, Max for maximum, and Scal. Sol. for scaling solution. The $\gamma_{e f f}$ index is defined by $\gamma_{\text {eff }}=2 /(3 A)$ where $A$ characterizes the power-law expansion $a \propto t^{A}$.

\begin{tabular}{|c|c|c|c|c|c|}
\hline$x_{*}$ & $y_{*}$ & $\phi$ & $V(\phi)$ & Eq. state & Stability \\
\hline 0 & 0 & $\phi_{*}$ & $V_{*}=0 \operatorname{Min}(V)$ & - & $\mathrm{S} / \mathrm{MD}$ \\
\hline 0 & 0 & $\phi_{*}$ & $V_{*}=0$ & - & $\mathrm{U} / \mathrm{MD}$ \\
\hline 0 & 1 & $\phi_{*}$ & $V_{*} \neq 0 \operatorname{Min}(V)$ & $\gamma_{\phi}=0$ & $\mathrm{~S} / \mathrm{SFD}$ \\
\hline 0 & 1 & $\phi_{*}$ & $V_{*} \neq 0 \operatorname{Max}(V)$. & $\gamma_{\phi}=0$ & $\mathrm{U} / \mathrm{SFD}$ \\
\hline 0 & 0 & $\phi_{\infty}$ & $V_{\infty}=0$ & - & $\mathrm{U} / \mathrm{MD}$ \\
\hline 0 & 1 & $\phi_{\infty}$ & $V_{\infty} \neq 0$ & $\gamma_{\phi}=0$ & $\mathrm{~S} / \mathrm{SFD}$ \\
\hline $0<x_{0}<1$ & $0<y_{0}<1$ & $\phi_{\infty}$ & $V \sim e^{-\lambda \phi}$ & $\gamma_{e f f}<\gamma$ & $\mathrm{S} /$ Scal. Sol., $\lambda^{2}>3 \gamma$ \\
\hline $0<x_{0}<1$ & $0<y_{0}<1$ & $\phi_{\infty}$ & $V \sim e^{-\lambda \phi}$ & $\gamma_{e f f}>\gamma$ & S/Scal. Sol., $\lambda^{2}<3 \gamma$ \\
\hline
\end{tabular}


TABLE III: The properties of the scaling, asymptotic behavior of the $\Gamma \propto \Gamma(\phi) H^{\delta}, \Pi=$ $-3 \zeta \rho^{\alpha} H^{2(1-\alpha)}$ model: In this table S stands for stable and Scal. Sol. stands for scaling solution. The $\gamma_{e f f}$ index is defined by $\gamma_{e f f}=2 /(3 A)$ where $A$ characterizes the power-law expansion $a \propto t^{A}$.

\begin{tabular}{|c|c|c|c|c|c|}
\hline$x_{*}$ & $y_{*}$ & $\phi$ & $V(\phi)$ & Eq. state & Stability \\
\hline $0<x_{0}<1$ & $0<y_{0}<1$ & $\phi_{\infty}$ & $V \sim e^{-\lambda \phi}, \Gamma(\phi) \sim V_{\infty}^{\frac{1-\delta}{2}}$ & $\gamma_{e f f}<\gamma$ & S/Scal. Sol., $\lambda^{2}>3 \gamma$ \\
\hline $0<x_{0}<1$ & $0<y_{0}<1$ & $\phi_{\infty}$ & $V \sim e^{-\lambda \phi}, \Gamma(\phi) \sim V_{\infty}^{\frac{1-\delta}{2}}$ & $\gamma_{e f f}>\gamma$ & S/Scal. Sol., $\lambda^{2}<3 \gamma$ \\
\hline
\end{tabular}

